Phylogeny, Ecology, and Heart Position in Snakes

Author(s): Gabriel E. A. Gartner, James W. Hicks, Paulo R. Manzani, Denis V. Andrade,

Augusto S. Abe, Tobias Wang, Stephen M. Secor, and Theodore Garland Jr.

Source: Physiological and Biochemical Zoology, Vol. 83, No. 1 (January/February 2010), pp. 4354

Published by: The University of Chicago Press

Stable URL: http://www.jstor.org/stable/10.1086/648509

Accessed: 28/08/2013 10:46

Your use of the JSTOR archive indicates your acceptance of the Terms \& Conditions of Use, available at http://www.jstor.org/page/info/about/policies/terms.jsp

JSTOR is a not-for-profit service that helps scholars, researchers, and students discover, use, and build upon a wide range of content in a trusted digital archive. We use information technology and tools to increase productivity and facilitate new forms of scholarship. For more information about JSTOR, please contact support@jstor.org. 


\section{Phylogeny, Ecology, and Heart Position in Snakes}

\author{
Gabriel E. A. Gartner ${ }^{1, \star}$ \\ James W. Hicks ${ }^{2, \dagger}$ \\ Paulo R. Manzani ${ }^{3, *}$ \\ Denis V. Andrade ${ }^{3,4, \$}$ \\ Augusto S. Abe Ab, $^{3,4}$ \\ Tobias Wang,"\# \\ Stephen M. Secor ${ }^{6, * *}$ \\ Theodore Garland Jr. ${ }^{1, \dagger \dagger}$ \\ ${ }^{1}$ Department of Biology, University of California, Riverside, \\ California 92521; ${ }^{2}$ Department of Ecology and Evolutionary \\ Biology, University of California, Irvine, California 92697- \\ 2525; ${ }^{3}$ Departamento de Zoologia, Universidade Estadual \\ Paulista, Rio Claro, São Paulo 13506-900, Brazil; ${ }^{4}$ Instituto \\ Nacional de Ciência e Tecnologia em Fisiologia Comparada; \\ ${ }^{5}$ Zoophysiology, Department of Biological Sciences, \\ University of Aarhus, Denmark; ${ }^{6}$ Department of Biological \\ Sciences, Box 870344, University of Alabama, Tuscaloosa, \\ Alabama 35487
}

Accepted 3/21/2009; Electronically Published 12/7/2009

Online enhancements: data file, appendixes.

\begin{abstract}
The cardiovascular system of all animals is affected by gravitational pressure gradients, the intensity of which varies according to organismic features, behavior, and habitat occupied. A previous nonphylogenetic analysis of heart position in snakes - which often assume vertical postures-found the heart located $15 \%-25 \%$ of total body length from the head in terrestrial and arboreal species but $25 \%-45 \%$ in aquatic species. It was hypothesized that a more anterior heart in arboreal species served to reduce the hydrostatic blood pressure when these animals adopt vertical postures during climbing, whereas an anterior heart position would not be needed in aquatic habitats, where the effects of gravity are less pronounced. We

*E-mail: ggart001@ucr.edu.

${ }^{\dagger}$ E-mail: jhicks@uci.edu.

${ }^{\ddagger}$ Present address: Universidade Estadual de Campinas-UNICAMP, Departamento de Zoologia-Instituto de Biologia, Campinas-SP 13083-970, Brazil; email: pmanzani@rc.unesp.br.

${ }^{\S}$ E-mail: denis@rc.unesp.br.

"E-mail: asabe@rc.unesp.br

${ }^{\#}$ E-mail: tobias.wang@biology.au.dk.

** E-mail: ssecor@biology.as.ua.edu.

${ }^{+\dagger}$ Corresponding author; e-mail: tgarland@ucr.edu.
\end{abstract}

Physiological and Biochemical Zoology 83(1):43-54. 2010. (C) 2010 by The University of Chicago. All rights reserved. 1522-2152/2010/8301-8173\$15.00 DOI: $10.1086 / 648509$ analyzed a new data set of 155 species from five major families of Alethinophidia (one of the two major branches of snakes, the other being blind snakes, Scolecophidia) using both conventional and phylogenetically based statistical methods. General linear models regressing $\log _{10}$ snout-heart position on $\log _{10}$ snout-vent length (SVL), as well as dummy variables coding for habitat and/or clade, were compared using likelihood ratio tests and the Akaike Information Criterion. Heart distance to the tip of the snout scaled isometrically with SVL. In all instances, phylogenetic models that incorporated transformation of the branch lengths under an Ornstein-Uhlenbeck model of evolution (to mimic stabilizing selection) better fit the data as compared with their nonphylogenetic counterparts. The bestfit model predicting snake heart position included aspects of both habitat and clade and indicated that arboreal snakes in our study tend to have hearts placed more posteriorly, opposite the trend identified in previous studies. Phylogenetic signal in relative heart position was apparent both within and among clades. Our results suggest that overcoming gravitational pressure gradients in snakes most likely involves the combined action of several cardiovascular and behavioral adaptations in addition to alterations in relative heart location.

\section{Introduction}

With the exception of body length, which varies by two orders of magnitude, the snake bauplan is conserved across all taxa (Greene and McDiarmid 2005). All snakes are limbless, elongate, and lack a pectoral girdle; their anatomy is elegantly modified to fit a tubular body plan (Greene 1997; Cohn and Tickle 1999). Although they might be viewed as occupying a relatively small region of "morphospace" with respect to basic body plan, snakes have radiated extensively in both number of species (>3,100 named; Uetz et al. 2007) and behavioral ecology. Even within the apparent restrictions associated with a limbless and elongate lifestyle, snakes have evolved to occupy almost all ecological niches, including fully aquatic and pelagic sea snakes, arboreal species that rarely if ever come to the forest floor, snakes that glide, and completely fossorial burrowers (Greene 1997; Martins et al. 2008).

Despite its evolutionary success, the snake body plan is nevertheless subject to constraint at several levels. Gravity, in particular, may significantly affect the cardiovascular function of snakes, which are in essence long fluid-filled tubes (Lillywhite 1987). In a vertical column of fluid (e.g., arteries and veins), gravity creates a vertical pressure gradient that increases with the height of the tube (i.e., $\rho g h$; where $\rho$ is the density of blood, $g$ is the acceleration due to gravity, and $h$ is the vertical height of the fluid column above or below a reference plane; in this 


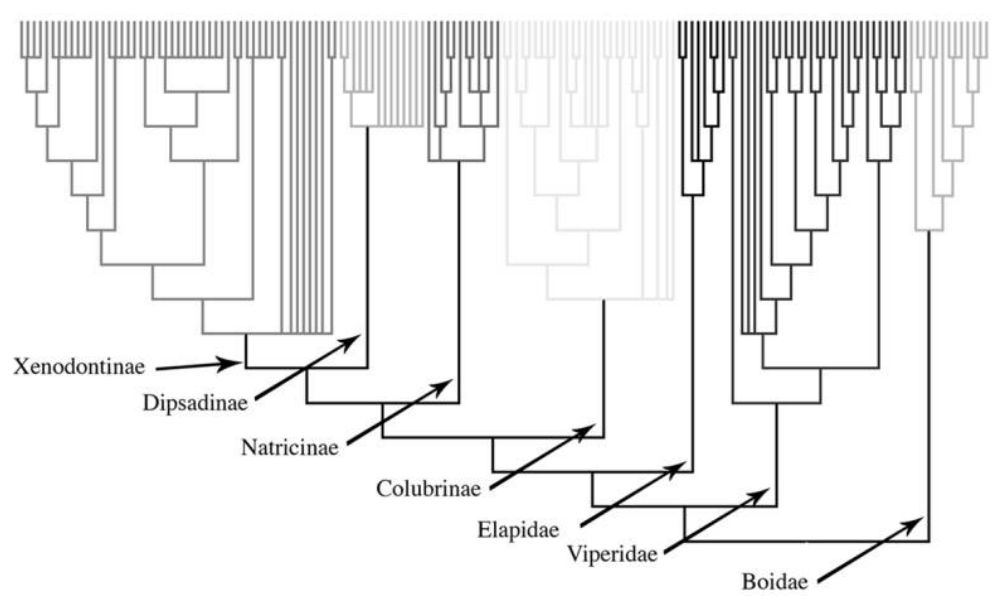

Figure 1. Phylogeny used for statistical analyses, with Pagel's (1992) arbitrary branch lengths. See Appendix B for details of tree construction and Appendix C for an electronic version (both appendixes are available in the online edition of Physiological and Biochemical Zoology).

case the heart). Gravitational pressure gradients have several physiological consequences. For example, increased gravitational pressure will distend the distal veins below the heart and may cause significant blood pooling and increased plasma leakage into the surrounding tissues. In addition, venous blood pooling will tend to reduce cardiac filling and cardiac output that if not compensated by an appropriate baroreceptor response can decrease arterial blood pressure and blood flow to the brain. Therefore, animals - particularly those that are upright or assume vertical postures-must find solutions to increased gravitational pressure gradients on the cardiovasuclar system. In the giraffe, for example, several mechanisms are known to work together to prevent edema in the lower extremities, including thick and impermeable capillary basement membranes, arterial-wall hypertrophy, and a prominent lymphatic system (Willamson et al. 1971; Nilsson et al. 1988; Hargens 1991).

For at least two reasons, snakes are an interesting group in which to study potential cardiovascular adaptations that counter gravitational pressure gradients. First, some species are completely terrestrial and therefore rarely encounter changes in gravity, whereas arboreal species frequently assume vertical postures while climbing, and aquatic species are less vulnerable to the effects of gravity. Second, the conservative body plan eliminates the confounding effects of appendages.

The utility of snakes as models to study the effects of gravity and adaptations of the cardiovascular system to gravitational pressure gradients has been previously reported (Lillywhite 1987, 1988; Seymour 1987). The most intriguing adaptive hypothesis to emerge from these studies is that heart position should correlate with behavioral ecology or habitat. Specifically, in nonaquatic snakes that frequently assume vertical postures, the height of the heart-head blood column should be reduced, thus reducing cardiac work. In contrast, aquatic species would have more centrally placed hearts because of the more dense and less gravity-stressing nature of the medium they occupy (water). Finally, terrestrial species would have a heart position intermediate between aquatic and arboreal forms (Seymour and Lillywhite 1976; Lillywhite 1987, 1988; Seymour 1987; Lillywhite and Henderson 1993; Lillywhite et al. 1996a). Although this general adaptive hypothesis seems reasonable, it must be compared with alternative explanations to describe the evolutionary origin and maintenance of traits (e.g., see Gould and Lewontin 1979; Greene 1986; Garland et al. 1993; Garland and Adolph 1994; Garland and Carter 1994; Rose and Lauder 1996; Clobert et al. 1998; Orzack and Sober 2001; Blomberg and Garland 2002; Garland et al. 2005).

In this article, we analyze heart position in 155 , primarily South American, snake species or subspecies to investigate the generality of the heart position/habitat hypothesis using both phylogenetic and nonphylogenetic statistical models. We account for phylogenetic "effects" (in the statistical sense) in two ways. The first is by use of a phylogenetic tree that is hierarchical (as shown in Fig. 1) versus one that has no hierarchical structure and appears as a "star" (one large polytomy). The hierarchical phylogeny, and alterations of it under an Ornstein-Uhlenbeck (OU) model of character evolution (see "Methods"), implies that related species will tend to be similar for the dependent variable of interest, in our case heart position. The second way that we allow for phylogenetic effects in statistical models is by coding major branches of the tree for our included species as different levels in a categorical factor (as shown in Fig. 1, we recognized seven different lineages or "clades"). By use of $\ln$ maximum likelihood ratio tests (LRTs), we can compare the fit of models that assume (1) a star phylogeny (implying no expected tendency for relatives to resemble each other), (2) the hierarchical phylogeny shown in Figure 1, or (3) the hierarchical phylogeny transformed under an OU model of evolution (see "Methods"). We also use LRTs to compare those three models with their counterparts that include the clade variable (which we can refer to as models 4,5 , and 6, respectively). Importantly, this approach is not the same as nested ANOVAs based on taxonomy rather than phylogeny per se (see review in Harvey and Pagel 1991). Those approaches could implement a star 
phylogeny with categorical variables to represent membership in different named groups at one or more taxonomic levels (e.g., order, family, genus) but could not incorporate the full branching structure either among groups at a given level or within a group.

We also apply the above-listed six models with a separate categorical variable that codes for variation in an "ecological" factor-namely habitat (Table 1: terrestrial, fossorial, arboreal, semiaquatic) — and compare their fit with LRTs. Finally, we use the Akaike Information Criterion (AIC) to compare the fit of nonnested models that include the habitat variable, the clade variable, or both. These AIC comparisons allow for simultaneous consideration of the statistical effects of both phylogeny and ecology on relative heart position. Our overall statistical approach is widely applicable to many questions in comparative biology (see also Huey et al. 2009; Swanson and Garland 2009).

\section{Methods}

\section{Heart Position, Habitat, and Body Size}

We gathered data on snake heart position in 155 taxa representing seven major families and subfamilies from both new material and museum specimens (Table 1; see App. A in the online edition of Physiological and Biochemical Zoology for data). Snakes came primarily from a data set collected in Brazil by P. R. Manzani and D. V. Andrade (Manzani 1995; $N=$ 120 species). The remaining species were measured by G. E. A Gartner ( $N=8$ species $)$ and S. M. Secor $(N=27$ species $)$. We used the mean snout-heart length (SHL) when multiple individuals of a species were measured (App. A). In general, only adult snakes were used. Heart distance was measured by making a ventral incision from the neck until reaching the heart, then measuring the distance from the tip of the snout to the top of the atria. Snout-vent length (SVL) was measured from the tip of the rostral scale to the cloaca on the ventral side of the animal. Note that in Seymour (1987, p. 90) "the distances [were] measured between the head (eye), heart and tip of tail." This difference in measurements is, more likely than not, negligible from a hydrostatic standpoint, as it is unlikely that the evolution of the circulatory system is driven by such minimal differences in pressure.

Snakes were categorized with respect to general habitat usage using literature accounts-primarily field guides and various works on localized snake faunas (e.g., Wright and Wright
1957) — and the authors' own observations and experiences with many of the included species. Fossorial species actively burrow and are found underground or in litter, and most possess obvious morphological adaptations for burrowing (e.g., Typhlops). Semiaquatic species are commonly found in water, where they often feed or flee from predators but frequently take to the shore to bask, reproduce, etc. (e.g., Nerodia). Arboreal species are often long and gracile in appearance and are most frequently encountered in trees or low-lying shrubs (e.g., Boiga, Corallus). Terrestrial species lack any obvious morphological adaptations to the terrestrial realm and thus cannot be easily classified into any of the other groups (e.g., most elapids, Pituophis, Drymarchon). When possible, habitat categories were chosen to reflect those of a previous study (Seymour 1987). Thus, the terrestrial group not only includes obviously terrestrial animals found away from water or trees but also those snakes occasionally found swimming or climbing (as most snakes appear to be able to swim and climb to some extent). Unlike in Seymour (1987), our data set included only one species that might be considered aquatic (Micrurus surinamensis), so it was coded as semiaquatic for purposes of statistical analyses.

\section{Phylogeny Construction}

We constructed a composite tree using phylogenetic hypotheses from previously published studies. We began with higher-level relationships uniting the major lineages of snakes and nested less inclusive groups (lower-level relationships) within this framework. Our initial intent was to use the best available phylogenetic estimate at each hierarchical level rather than combining a number of phylogenies for a particular group. Most published trees, however, contained only a few taxa of interest for any particular group, and so we were often forced to use multiple trees-each of which may have employed different characters and methods in their analyses-to place particular taxa into our tree.

When a number of trees were available for a given group, we followed the methods of de Queiroz and Rodríguez-Robles (2006): maximum likelihood trees were preferred over those obtained by other methods (e.g., parsimony), and strict consensus trees were used when available. If a lower-level group had multiple trees available to choose from, then we used the number of characters and the number of taxa to differentiate

Table 1: Families or subfamilies included in the data set and their habitat distributions

\begin{tabular}{lcccccccc}
\hline & Xenodontinae & Dipsadinae & Natricinae & Colubrinae & Elapidae & Viperidae & Boidae & Total \\
\hline Terrestrial & 35 & 6 & 1 & 11 & 2 & 22 & 6 & 83 \\
Fossorial & 4 & 2 & $\ldots$ & 2 & 5 & $\ldots$ & 1 & 14 \\
Arboreal & 5 & 6 & $\ldots$ & 15 & $\ldots$ & 5 & 3 & 34 \\
Semiaquatic & 7 & $\ldots$ & 11 & $\ldots$ & 0 & 2 & 3 & 23 \\
Aquatic & $\ldots$ & $\ldots$ & $\ldots$ & $\ldots$ & $1^{\text {a }}$ & $\ldots$ & $\ldots$ & $1^{\text {a }}$ \\
\cline { 2 - 9 } Total & 51 & 14 & 12 & 28 & 8 & 29 & 13 & 155 \\
\hline
\end{tabular}

${ }^{a}$ The aquatic Micrurus surinamensis was recoded as semiaquatic for purposes of statistical analyses. 
Table 2: Allometry of snout-heart length in relation to snout-vent length

\begin{tabular}{|c|c|c|c|c|c|c|c|}
\hline Model & Slope & SE & $y$-intercept & SE & $r^{2}$ & $\begin{array}{l}\text { In Maximum } \\
\text { Likelihood }\end{array}$ & AIC \\
\hline \multicolumn{8}{|l|}{ Conventional: } \\
\hline Least squares & 1.027 & .0489 & -.729 & .1375 & .742 & 118.791 & -231.6 \\
\hline Reduced major axis & 1.193 & & -1.191 & & & & \\
\hline \multicolumn{8}{|l|}{ Phylogenetic: } \\
\hline Least squares & .926 & .0351 & -.405 & .1204 & .820 & 168.798 & -331.6 \\
\hline Reduced major axis & 1.023 & & -.679 & & & & \\
\hline Regression with OU transform ${ }^{a}$ & .937 & .0362 & -.447 & .1061 & .814 & 174.916 & -341.8 \\
\hline
\end{tabular}

${ }^{a}$ REML estimate of $d=0.7306$.

among them. For instance, if two trees had similar numbers of taxa, we chose the tree with the greater number of characters (and vice versa).

In a few instances, the amount of clade support (i.e., bootstrap values) influenced our decision on tree selection. In those cases where no phylogenetic hypotheses could be found (particularly a problem for species-level relationships among the Xenodontinae) or where there was particularly weak nodal support, we collapsed clades to maintain a more conservative approach to our analysis.

Figure 1 shows the topology of the final tree and indicates the seven major clades identified in statistical analyses. Details of tree construction can be found in Appendix B in the online edition of Physiological and Biochemical Zoology. For statistical analyses, branch lengths were set by the arbitrary method of Pagel (1992), as shown in Figure 1, using the DOS PDTREE program (Garland et al. 1999; Garland and Ives 2000). Appendix $\mathrm{C}$ in the online edition of Physiological and Biochemical Zoology presents the tree in a standard electronic format.

\section{Statistical Analyses}

SVL and SHL were $\log _{10}$ transformed before analyses. To test and quantify phylogenetic signal, we used the methods of Blomberg et al. (2003).

We then described the simple allometry of snout-heart position in relation to SVL in the entire data set $(N=155)$ by use of ordinary least squares (OLS) linear regression and reduced major axis (RMA), with both conventional (i.e., nonphylogenetic or assuming a star phylogeny) and phylogenetic versions, using the DOS PDTREE program. PDTREE employs phylogenetically independent contrasts, which yields estimates that are the same as from a phylogenetic generalized least squares analysis (PGLS; Garland et al. 2005; Lavin et al. 2008). However, PDTREE provides certain statistics that are not currently available in most programs for PGLS. It is well known that OLS slopes will underestimate the true scaling relation when the independent variable (in this case, $\log _{10}$ SVL) contains "measurement error," and if the amount of such error is not known, then the RMA slope often gives a reasonable estimate (Rayner 1985; Warton et al. 2006; Ives et al. 2007). To obtain the likelihood of the alternative models, we used the Matlab Regressionv2.m program developed by A. R. Ives and T. Gar- land Jr. (Lavin et al. 2008; for examples of applications, see Buchwalter et al. 2008; Jeffery et al. 2008; Warne and Charnov 2008; Huey et al. 2009; Swanson and Garland 2009).

Next, we examined the effects of body size (SVL), clade, and ecology using conventional multiple regressions with dummy variables that code for clade membership and habitat category (i.e., ANCOVA with parallel slopes). Formally, a "clade" is defined as a monophyletic group of organisms, including the ancestor and all descendant species. Practically, few if any comparative studies can include all members of a given clade because of extinctions and/or inaccessibility of living representatives. In this article, we use "clade" to refer to all of the species included in the available data set that are members of a formal clade (e.g., Colubrinae, Viperidae, Elapidae). For analyses, Re-

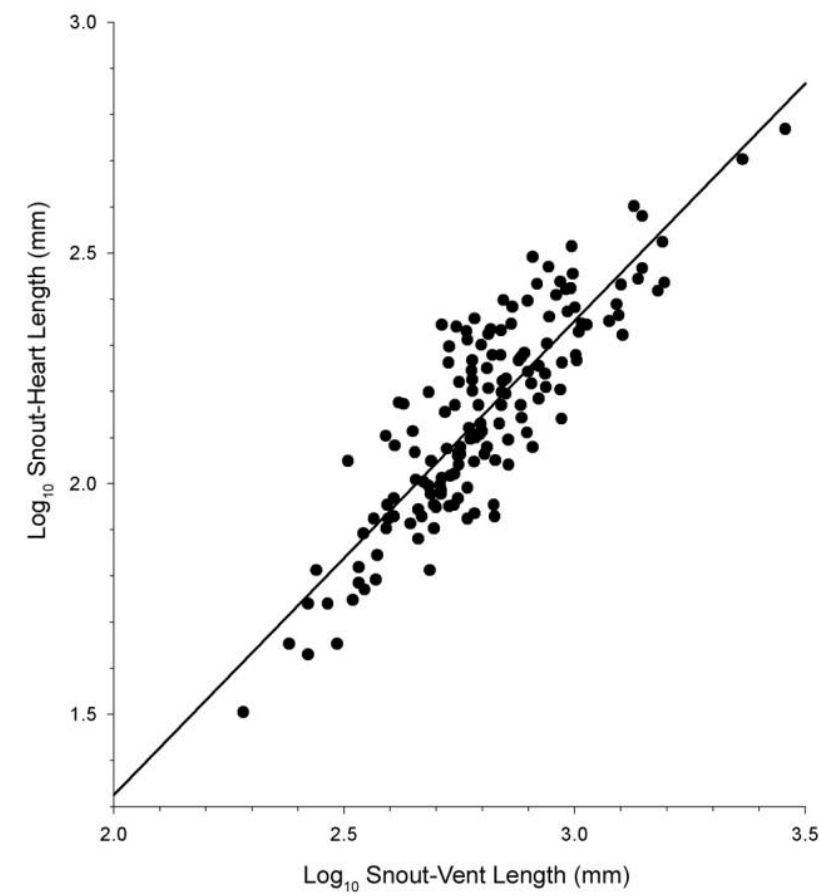

Figure 2. Isometry (slope not statistically different from 1.00) of snoutheart length in relation to snout-vent length for 155 species or subspecies of snakes. Line is conventional (nonphylogenetic) least squares linear regression (slope $=1.027, \mathrm{SE}=0.0489$; see Table 2 for this and alternatives). 
Table 3: Alternate regression models for predicting heart position of snakes

\begin{tabular}{|c|c|c|c|c|c|c|c|c|c|c|}
\hline \multirow[b]{2}{*}{ Model } & \multicolumn{2}{|c|}{$\begin{array}{l}\text { Conventional } \\
\text { (OLS) }\end{array}$} & \multicolumn{2}{|c|}{$\begin{array}{l}\text { Phylogeny } \\
\text { (PGLS) }\end{array}$} & \multicolumn{2}{|c|}{$\begin{array}{l}\text { Phylogeny with } \\
\text { OU Transform } \\
\text { (RegOU) }\end{array}$} & \multirow[b]{2}{*}{ Mean SE } & \multirow{2}{*}{$\begin{array}{l}\text { SE of } \\
\text { the } \\
\text { Estimate }\end{array}$} & \multirow{2}{*}{$\begin{array}{l}r^{2} \text { for } \\
\text { Model }\end{array}$} & \multirow{2}{*}{$\begin{array}{l}\text { REML } \\
\text { Estimate } \\
\text { of } d \text { (OU } \\
\text { Transform) }\end{array}$} \\
\hline & $\ln \mathrm{ML}$ & AIC & $\ln \mathrm{ML}$ & AIC & $\ln \mathrm{ML}$ & AIC & & & & \\
\hline SVL (simple allometry) & 118.791 & -231.6 & 168.798 & -331.6 & 174.916 & -341.8 & $6.210 \mathrm{E}-03$ & .07880 & .8140 & .7306 \\
\hline SVL + habitat & 126.673 & -241.3 & 178.459 & -344.9 & 184.909 & -355.8 & $5.567 \mathrm{E}-03$ & .07462 & .8367 & .7356 \\
\hline SVL + clade & 187.324 & -356.6 & 170.298 & -322.6 & 193.673 & -367.3 & $5.114 \mathrm{E}-03$ & .07151 & .8602 & .3024 \\
\hline $\begin{array}{l}\text { SVL }+ \text { clade }+ \text { habitat } \\
\text { SVL }+ \text { clade }+\end{array}$ & 205.625 & -387.3 & 180.134 & -336.2 & 209.749 & -393.5 & $4.234 \mathrm{E}-03$ & .06507 & .8936 & .1975 \\
\hline “arboreal” & 204.655 & -389.3 & 179.974 & -339.9 & 209.075 & -396.2 & $4.210 \mathrm{E}-03$ & .06489 & .8923 & .2034 \\
\hline
\end{tabular}

Note. $\mathrm{ML}=$ maximum likelihood. "Conventional (OLS)" indicates ordinary least squares (multiple) regression, which is mathematically equivalent to assuming a "star" phylogeny with no hierarchical structure. "Phylogeny" indicates generalized least squares analysis (PGLS), which is mathematically equivalent to phylogenetically independent contrasts. "Phylogeny with OU Transform (RegOU)" is a regression model in which the residuals are modeled as an Ornstein-Uhlenbeck process. RegOU models contain one more parameter than their OLS or PGLS counterparts, so whether a RegOU model fits the data significantly $(P<0.05)$ better can be tested by comparing twice the difference in ln likelihoods with the value 3.841 (the ninety-fifth percentile of the distribution of $\chi^{2}$ with 1 df). Similar In likelihood ratio tests can be used to compare models within the OLS, PGLS, or RegOU columns when one is a nested subset of the other (e.g., SVL + habitat vs. SVL but not SVL + habitat vs. SVL + clade). The AIC (see "Methods") can be used to compare any models, with smaller (more negative) values indicating a better fit. As a rule of thumb, models whose AIC is $<2$ units larger than the best model can also be said to have substantial support. See "Methods" and Lavin et al. (2008) for further explanation. For the models listed in the bottom row (SVL + clade + "arboreal"), the arboreal variable was always highly significant (all $P<0.0001$ ), and the partial regression coefficient was always positive, indicating that heart position is more posterior than for nonarboreal species.

gressionv2.m automatically recoded clade as a series of six 0 1 dummy variables.

Our simplest model used only SVL as an independent variable and hence was just a linear regression. Subsequent models added additional variables along with SVL (e.g., SVL + clade or SVL + habitat). Our most complex or "full" model included SVL, clade, and habitat. Based on inspection of the partial regression coefficients for that most complex model (see "Results"), it was apparent that the major effect of habitat was that arboreal animals were different from all three other habitat types. Therefore, we ran one additional model that considered SVL, clade, and only arboreal animals as a distinct category.

All analyses were then repeated using PGLS ANCOVA models with the Regressionv2.m program. Finally, we implemented phylogenetic ANCOVAs with a branch-length transformation parameter based on the OU model of evolution, termed "RegOU" (Lavin et al. 2008). The OU process has been suggested as a way to mimic the effects of stabilizing selection (e.g., see Felsenstein 1988; Garland et al. 1993; Blomberg et al. 2003; Halsey et al. 2006; Lavin et al. 2008; see also Martins and Hansen 1997). The statistical procedure begins with a userspecified phylogenetic tree (as shown in Fig. 1), then moves the internal nodes of the tree up and down, thus simultaneously stretching and contracting the branch lengths above and below the nodes. Small values of the OU transformation parameter (d) yield trees that are more starlike (i.e., long terminal branches and short internode branches near the root), whereas values of $d$ greater than unity yield trees that are even more hierarchical than the original tree. A $d$ value of exactly unity yields the original tree. The regression model in question is fitted using the entire range of stretched and compressed trees. The tree that yields the lowest mean squared error (residual variance) has the highest likelihood, and it is used to compute regression statistics. All of the multiple regression analyses were performed in Matlab version 7.0 using Regressionv2.m (Lavin et al. 2008). For the conventional (nonphylogenetic) multiple regressions, analyses were also run in SPSS for Windows version 11.5 as a verification, and results were identical to those of Regressionv2.m. The fit of all alternate models considered was compared using the AIC, computed in the smaller-is-better form: AIC $=(-2 \times \ln$ ML likelihood $)+(2 \times$ no. parameters $)$.

AIC is particularly well suited to situations such as this, where numerous nonnested models are being compared (e.g., see Lavin et al. 2008). As a rule of thumb, models whose AIC is $<2$ units larger can also be said to have substantial support, whereas a difference of 4-7 indicates considerably less support, and a difference $>10$ indicates essentially no support (Burnham and Anderson 2002, p. 70). Where one model was a nested subset of the other, we compared them by ln maximum likelihood ratio tests (LRTs), where twice the difference in ln likelihoods is assumed to be distributed asymptotically as a $\chi^{2}$ distribution with degrees of freedom equal to the difference in the number of parameters in the two models. If an LRT and/ or comparison of the AIC indicates that the phylogenetic version of the model is better than the nonphylogenetic version, then one can conclude that "phylogenetic signal"-the tendency for similar species to resemble one another (Blomberg and Garland 2002; Blomberg et al. 2003) - is present in the residuals of the nonphylogenetic model. From a more general perspective, if the clade variable is statistically significant in a model, then phylogenetic position is also important in predicting the dependent variable (i.e., heart position in this article) after controlling statistically for other independent variables in the model.

\section{Results}

SVL ( $\log _{10}$ transformed) showed relatively low $(K=0.265$; cf. Blomberg et al. 2003) but statistically significant $(P<0.001)$ 
phylogenetic signal. Once corrected for its association with $\log _{10}$ SVL, $\log _{10}$ heart position showed higher signal $(K=0.580$, $P<0.001)$.

Among all taxa, heart position scales isometrically with body size (Table 2; Fig. 2). The 95\% confidence interval (CI; 0.9311.124) about the OLS regression slope (1.027) includes unity, although, as must be the case, the RMA slope is higher (RMA slope $=1.193 ; \mathrm{RMA}=\mathrm{OLS} / r)$. The 95\% CI $(0.856-0.995)$ about the PGLS slope (0.926) excludes unity, but again, the RMA slope is higher (1.023). The phylogenetic regression with an OU transform (RegOU) is the best-fitting model (based on likelihood and AIC; Table 2) and has a slope of 0.937 with a 95\% CI of $0.865-1.008$.

Alternate models that include clade and/or habitat are presented in Table 3. Based on the AIC values (smaller is better), the relative fit of the models with various independent variables is the same for conventional OLS and RegOU models, improving in the following order: SVL (simple allometry), SVL + habitat, SVL + clade, SVL + clade + habitat, SVL + clade + "arboreal" (i.e., a single category of habitat vs. all others). However, for all models, the RegOU versions (which contain one more parameter) are significantly better than their OLS counterparts based on ln likelihood ratio (LR) tests, with the largest $P$ value being 0.0041 for the model that includes SVL + clade + habitat. For both OLS and RegOU models, the best-fitting model, based on lowest AIC, includes SVL, clade, and a single dummy variable for arboreal snakes (rather than the set of three dummy variables to recognize all four habitat categories). This emphasizes that, for our data set, the major habitat effect on snake heart position is that arboreal snakes have more posteriorly positioned hearts (see partial regression coefficients for the full models in Table 4).

Table 3 also presents PGLS models, which incorporate the phylogeny with untransformed branch lengths, as shown in Figure 1. In all cases, these models are significantly worse than their RegOU counterparts (which contain one additional parameter), based on LR tests (largest $P=0.0005$ ). Therefore, we defer further consideration of these models to the "Discussion."

\section{Discussion}

We used a statistical approach that incorporates phylogenetic information to develop models that address whether ecological or historical factors (or a combination of the two) most affect relative heart position in snakes and to test for the presence of phylogenetic signal in this trait. We determined which version of a given model-OLS, PGLS, or RegOU—better fit the data by use of ln LRTs and by comparing AIC values.

Our general findings were that habitat, clade membership, and phylogenetic position within clades (and/or interclade relations) all accounted for some of the variation in relative heart position. The fact that the RegOU models fit better than the nonphylogenetic models even when clade is included as a factor (Table 3) means that the hierarchical structure within and/or among clades reflects some of the resemblance among related species (i.e., phylogenetic signal) in relative heart position. With respect to habitat, arboreal snakes had the most posteriorly placed hearts relative to SVL; with respect to clade, the Viperidae had the most posterior hearts (Fig. 3; Table 4).

Table 4: Full model including habitat and clade variables to predict $\log _{10}$ snout-heart length (mm), analyzed by conventional multiple regression and phylogenetically with an OU transform

\begin{tabular}{|c|c|c|c|c|c|c|c|c|c|c|}
\hline \multirow[b]{2}{*}{ Variable } & \multicolumn{5}{|c|}{ Conventional (OLS) } & \multicolumn{5}{|c|}{ Phylogenetic with OU Transform (RegOU) } \\
\hline & Coefficient & SE & $F$ & df & $P$ & Coefficient & SE & $F$ & df & $P$ \\
\hline$y$-intercept & -.6465 & .0964 & 44.94 & & & -.5746 & .0968 & 35.26 & & \\
\hline $\log _{10}$ SVL $(\mathrm{mm})$ & .9694 & .0331 & 858.82 & 1,144 & .0039 & .9454 & .0333 & 806.77 & 1,144 & $<.0001$ \\
\hline Arboreal & .0914 & .0148 & 38.14 & 1,144 & $<.0001$ & .0879 & .0155 & 32.06 & 1,144 & $<.0001$ \\
\hline Fossorial & .0191 & .0217 & .77 & 1,144 & .3813 & .0199 & .0230 & .75 & 1,144 & .3879 \\
\hline Semiaquatic & .0225 & .0196 & 1.31 & 1,144 & .2537 & .0156 & .0227 & .48 & 1,144 & .4895 \\
\hline Xenodontinae & -.0049 & .0174 & .08 & 1,144 & .7789 & -.0094 & .0224 & .18 & 1,144 & .672 \\
\hline Dipsadinae & .0691 & .0232 & 8.87 & 1,144 & .0034 & .0564 & .0307 & 3.37 & 1,144 & .0685 \\
\hline Natricinae & -.0448 & .0297 & 2.28 & 1,144 & .1335 & -.0483 & .0366 & 1.74 & 1,144 & .1892 \\
\hline Boidae & .1226 & .0235 & 27.14 & 1,144 & $<.0001$ & .1314 & .0303 & 18.81 & 1,144 & $<.0001$ \\
\hline Elapinae & .0718 & .0301 & 5.70 & 1,144 & .0183 & .0666 & .0355 & 3.52 & 1,144 & .0627 \\
\hline Viperidae & .2153 & .0187 & 132.27 & 1,144 & $<.0001$ & .2079 & .0229 & 82.49 & 1,144 & $<.0001$ \\
\hline Habitat & & & 12.79 & 3,144 & $<.0001$ & & & 10.71 & 3,144 & $<.0001$ \\
\hline Clade & & & 42.47 & 6,144 & $<.0001$ & & & 25.09 & 6,144 & $<.0001$ \\
\hline
\end{tabular}

Note. For the habitat variable, terrestrial is arbitrarily chosen as the base group for comparison. For the clade variable, Colubridae is arbitrarily chosen as the base group for comparison. Thus, all coefficients and significance levels for the individual dummy variables within these categorical variables are relative to those base groups. Overall tests for habitat and clade are at the bottom. For the OLS model, rate of evolution (MSE) $=$ 0.004438 , SE of estimate $(\mathrm{SEE})=0.06620$, model $r^{2}=0.9160$, ln maximum likelihood of model $=205.625$, AIC $=-387.250$, AIC $\mathrm{c}=-385.053$. For the RegOU model, MSE $=0.004234$, SEE $=0.06507$, model $r^{2}=0.8936$, REML estimate of OU transformation parameter $(d)=0.1975$, ln maximum likelihood of model $=209.749$, AIC $=-393.499, \mathrm{AIC}_{\mathrm{c}}=-390.917$. 
In all cases, the RegOU versions of models performed significantly better (based on LR tests) than their OLS or PGLS counterparts. The relative fit of the PGLS models, as shown in Table 3, reveals an interesting situation. The PGLS models incorporate the phylogeny with branch lengths as shown in Figure 1 and do not allow any branch-length transformations to improve the fit of the statistical model to the data. As compared with OLS models, their PGLS counterparts had much higher likelihoods when the clade variable was not in the model but lower likelihoods when it was included. This reflects a tradeoff in the sense that the phylogenetic signal (the tendency for related species to resemble each other; Blomberg and Garland 2002) present in the residuals can be apportioned either generally throughout the tree or among the specified clades but not both, given the branch lengths shown in Figure 1. However, when the branch lengths are allowed to vary to optimize fit in the RegOU models, the nodes are pulled toward the root (estimated $d$ value $\sim 0.2$, where $0=$ a star and $1.0=$ the original tree), thus making the tree more starlike than shown in Figure 1 , and models that include clade are much better (difference in AIC $=11.5-40.4$ ) than those that do not. In these RegOU models that include clade, phylogenetic signal thus exists both among clades and among species within clades (or in the form of related clades resembling each other).

The importance of estimating a branch-length transformation parameter simultaneously with estimating parameters in a phylogenetic regression model was first emphasized by Grafen (1989; see review in Lavin et al. 2008). Similarly, Garland et al. (1992) emphasized the importance of various diagnostics and possible transformations of branch lengths when implementing phylogenetically independent contrasts (see also Díaz-Uriarte and Garland 1998). These points are now well accepted in the comparative-method literature (e.g., Martins and Hansen 1997; Freckleton et al. 2002; Halsey et al. 2006; Duncan et al. 2007), and our study provides another clear example of how analyses can be improved by adding this flexibility. Moreover, debates about the importance of ecology versus phylogeny can be addressed statistically by comparison of a range of models in between a star and the original starter tree (e.g., Fig. 1) while simultaneously testing the effects of including ecological (e.g., habitat) and/or phylogenetic (clade membership) variables in alternate models (see also Huey et al. 2009; Swanson and Garland 2009).

In our study, the single best predictor of heart position was body size $\left(\log _{10} \mathrm{SVL}\right)$, which is unsurprising given the large size range in the data set (Tables 2, 4; Figs. 2, 3). With body size in the model, clade membership (see Fig. 1 for the seven clades identified for analyses) appeared to be a significantly better predictor of heart position than habitat (four categories identified) for both nonphylogenetic (AIC values of -356.6 for clade vs. -241.3 for habitat) and RegOU models ( -367.3 for clade vs. -355.8 for habitat). Moreover, those same AIC values indicate the clear superiority of the phylogenetic RegOU models as compared with the nonphylogenetic OLS models. Thus, relative heart position of snakes varies in relation to both differences among the major branches of the phylogenetic tree and
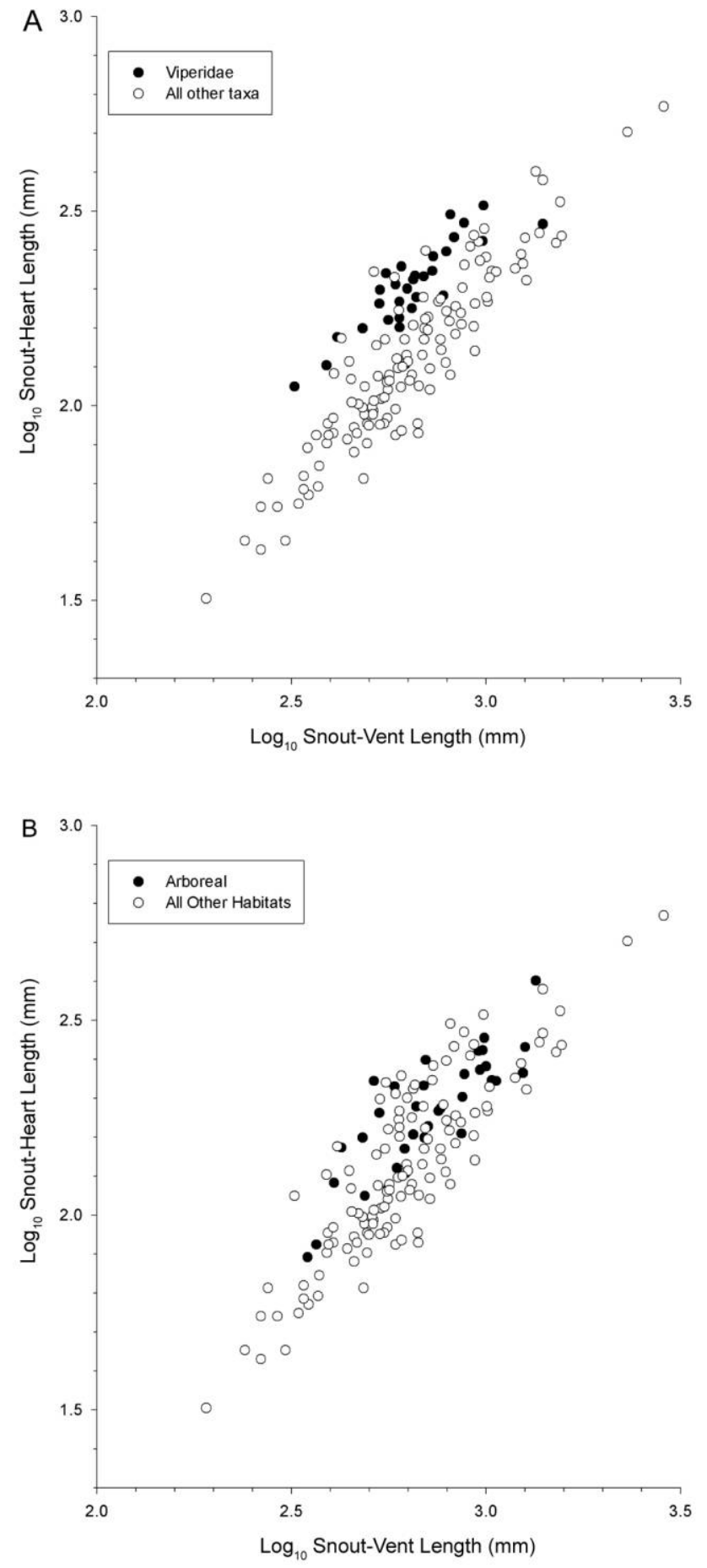

Figure 3. Snout-heart length in relation to snout-vent length for 155 taxa of snakes, indicating the clade that is most different ( $A$, Viperidae) and the habitat classification $(B$, arboreal) that is most different from other snakes.

the detailed hierarchical structure of the tree. Stated another way, phylogenetic signal (sensu Blomberg and Garland 2002; Blomberg et al. 2003) is apparent both within and among clades (see also above). The best models, however, incorporated both clade and habitat on a hierarchical tree, and the single best 


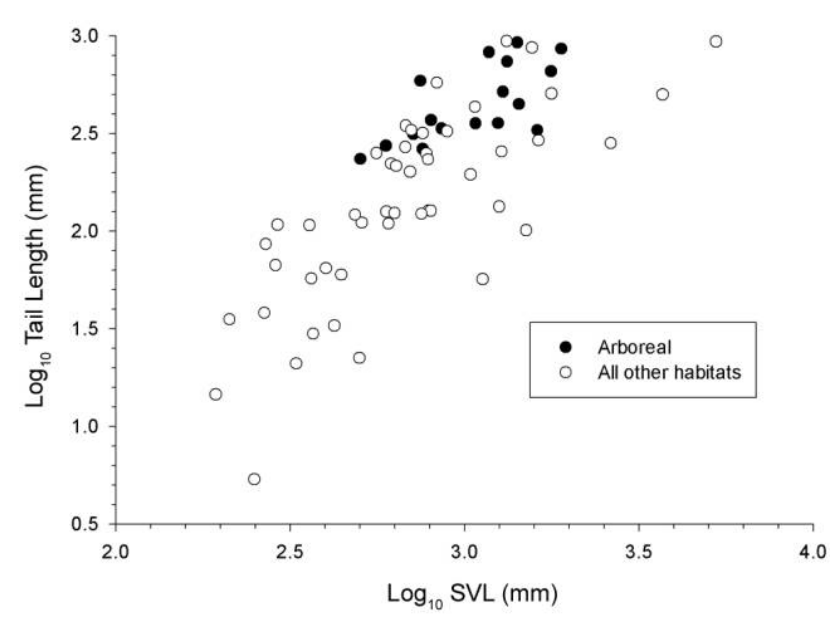

Figure 4. Tail length versus snout-vent length from a published data set for 65 South American taxa (Martins and Oliveira 1998). Note that arboreal species generally have longer tails (see text).

model included clade and only one aspect of habitat-inclusion in the arboreal category.

It is clear that both phylogeny and habitat are important in predicting heart position. For instance, in viperids, Seymour (1987) noted that the heart is generally shifted posteriorly (perhaps inherited from their common ancestor), but in arboreal members of the clade, the heart is positioned more anteriorly than in terrestrial members, which Seymour interpreted as an adaptation. Given their unusual heart positions, we also analyzed the viperids alone ( $N=29$ species), for which three habitat categories are represented (fossorial snakes are lacking). The ln likelihoods of the OLS, PGLS, and RegOU ANCOVA models were 45.0929, 44.8680, and 47.3945, respectively. Based on likelihood ratio tests, the RegOU model (estimated $d=$ $0.4630)$ is significantly better than either the OLS ( $P=$ $0.0319)$ or PGLS model $(P=0.0246)$. The effect of habitat was not statistically significant in any of the models $(P>0.5$ for the RegOU model).

For completeness, we also analyzed the Xenodontinae $(N=51)$ and Colubrinae $(N=28)$ alone. Results for Xenodontinae were similar to those for viperids; that is, RegOU was the best-fitting model, and habitat was not significant $(P>$ 0.5 for the RegOU model). For Colubrinae, the conventional OLS model was best, and habitat was highly significant $(P<$ 0.0001 ), with the effect attributable to arboreal species having hearts placed more posteriorly (partial regression coefficient $=$ $0.1074, P<0.0001)$. Thus, the habitat effect within Colubrinae alone is consistent with that shown in the overall analysis of all species (Table 4; Fig. 3).

\section{Alternate Functional Hypotheses for Variation in Heart Position}

The anterior heart position of arboreal snakes is hypothesized to reduce the cardiac work required to lift the blood to the head when in the vertical orientation (Seymour 1987; Lillywhite
1988). Alternatively, Badeer (1998) hypothesized that heart position is optimized for cardiac-filling pressures and is related to the compliance of the vessels above and below the heart. Briefly, this alternative view is based on the physical principles that determine the hydrostatic indifference point (HIP) in a vertically oriented vascular system (Wagner 1886; Clark et al. 1934; Gauer and Thron 1965). The HIP is a unique reference within the venous circulation where blood pressure is unaffected by vertical orientation (Gauer and Thron 1965; Buckner et al. 1999). HIP is determined by in vivo compliance of the veins above and below the heart. In the upright position, if the dependent veins (vessels below the heart) are highly compliant relative to the vessels above the heart, then the venous HIP will shift below heart level (Gauer and Thron 1965). The inferior location of the HIP results in a reduction in venous return and decreased cardiac-filling pressure (Buckner et al. 1999; Jarvis et al. 2007). Conversely, reducing the compliance (stiffening) of the dependent vessels can raise the HIP above heart level (Gauer and Thron 1965) and increase cardiac-filling pressures. The colocalization of the HIP and heart ensures that cardiac filling remains relatively stable despite changes in vertical orientation (Buckner et al. 1999). Based on these fundamental hemodynamic principles, Badeer (1998) hypothesized that the position of the heart in snakes should be correlated with the HIP.

Several studies have shown that arboreal snakes have less compliant caudal vessels compared with other terrestrial and aquatic species. In these species, the integument is tightly coupled to the underlying tissues, which will help prevent venous pooling in the upright position (Lillywhite 1993, 1996; Lillywhite et al. 1996b). Interestingly, this antigravity feature of the integument is similar to the "skin and fascial antigravity suit" seen in the legs of giraffes (Hargens et al. 1987), thus providing an apparent example of convergent evolution in function. In snakes, a more posterior location of the heart will also act in preventing blood from pooling below the heart when adopting an upright position (see the preceding discussion about HIP). Given that both decreased compliance (Lillywhite 1993, 1996; Lillywhite et al. 1996b) and posterior heart location (this study) are prevalent among arboreal species, this seems to indicate venous pooling and the associated risk of edema formation as an important gravitational stressor. Thus, previous adaptive scenarios placing the heart of arboreal snakes closer to the head may have overweighted the importance of ensuring an adequate blood supply to the head in detriment of the importance of venous return from the regions below the heart. Obviously, ensuring an adequate blood flow-above and below the heartis important both on the arterial and venous side, and this is only made possible by the action of an orchestrated suite of cardiovascular and behavioral adaptations rather than to alterations in heart location alone. This, combined with differences in the intensity of the gravitational stress imposed by different habitats, may help to explain the absence of any expected correlation between heart location and habitat for aquatic and terrestrial species. Future studies should address this question in addition to determining the compliance and 
Table 5: ANCOVA of snake heart position with $\log _{10}$ estimated total length as the covariate

\begin{tabular}{|c|c|c|c|c|c|c|c|c|c|c|}
\hline \multirow[b]{2}{*}{ Model } & \multicolumn{2}{|c|}{$\begin{array}{l}\text { Conventional } \\
\text { (OLS) }\end{array}$} & \multicolumn{2}{|c|}{$\begin{array}{l}\text { Phylogeny } \\
\text { (PGLS) }\end{array}$} & \multicolumn{2}{|c|}{$\begin{array}{l}\text { Phylogeny with } \\
\text { OU Transform } \\
\text { (RegOU) }\end{array}$} & \multirow{2}{*}{$\begin{array}{l}\text { Mean } \\
\text { Squared } \\
\text { Error }\end{array}$} & \multirow{2}{*}{$\begin{array}{l}\text { SE of } \\
\text { the } \\
\text { Estimate }\end{array}$} & \multirow{2}{*}{$\begin{array}{l}r^{2} \text { for } \\
\text { Model }\end{array}$} & \multirow{2}{*}{$\begin{array}{l}\text { REML } \\
\text { Estimate } \\
\text { of } d \text { (OU } \\
\text { Transform) }\end{array}$} \\
\hline & $\ln \mathrm{ML}$ & AIC & $\ln \mathrm{ML}$ & AIC & $\ln \mathrm{ML}$ & AIC & & & & \\
\hline $\begin{array}{l}\text { Total length + clade + } \\
\text { "arboreal" }\end{array}$ & 204.655 & -389.3 & 179.974 & -339.9 & 209.075 & -396.2 & $4.210 \mathrm{E}-03$ & .06489 & .8923 & .2034 \\
\hline$P$ for clade & $<.0001$ & & .7890 & & $<.0001$ & & & & & \\
\hline$P$ for arboreal & .0575 & & .3469 & & .0846 & & & & & \\
\hline
\end{tabular}

Note. $\mathrm{ML}=$ maximum likelihood. See note to Table 3 . The partial regression coefficient for the arboreal dummy variable was always positive, indicating that heart position is more posterior than for nonarboreal species.

the HIP of snakes from a variety of habitats, thus allowing direct tests of the hypothesis that heart position is correlated with HIP.

\section{Variation in Tail Length as a Possible Confounding Factor}

Seymour (1987) grouped snakes into six categories-arboreal, terrestrial, aquatic, semiaquatic, fossorial, and Viperidae-then analyzed heart position as a percent of total length (measured as eye to tail tip). ANOVA indicated highly significant group differences, with arboreal having the most anteriorly placed hearts (Table 6). In contrast, we analyzed SHL by ANCOVA with SVL as the covariate and found that arboreal snakes have more posteriorly placed hearts (Fig. 3; Table 4). One possible explanation for this discrepancy is that arboreal snakes in our data set tend to have relatively long tails $(\mathrm{H}$. B. Lillywhite, personal communication). Indeed, some previous studies have found that arboreal snakes often do have relatively long tails (Goldsmith 1984; Guyer and Donnelly 1990; Lillywhite and Henderson 1993).

In an attempt to test this proposition, we did the following. First, we analyzed a separate, published data set for 65 South American taxa (Martins and Oliveira 1998) that also measured SVL (as in our study) rather than total length and also reported tail length as a percent of total length. Tail length as a percentage of total length was given as a range of values, so we used the upper end of the range to compute absolute tail length for their data set. We then computed SVL by subtraction. Figure 4 shows that, for their data set, arboreal snakes do indeed tend to have relatively long tails. Conventional ANCOVA of their data yields the following equation:

$$
\begin{aligned}
& \log _{10} \text { total length }=0.04634+(1.01654 \\
& \left.\times \log _{10} S V L\right)+(0.06242 \times \text { arboreal })
\end{aligned}
$$

where "arboreal" is a dummy variable that is 1 for arboreal species and 0 for all others. Second, we used that equation to compute a $\log _{10}$ total length for all 155 species in our data set. Third, we analyzed the data with Regressionv2.m, mimicking the analyses presented in the bottom row of Table 3, but with $\log _{10}$ total length as the covariate rather than $\log _{10}$ SVL. In these analyses, the partial regression coefficient for the arboreal dummy variable was always positive, thus again indicating that arboreal snakes have hearts placed more posteriorly (unlike in the analyses of Seymour 1987), although the effect was not statistically significant (two-tailed $P=0.0846$ for the bestfitting RegOU model; see Table 5).

Table 6 shows the mean values from Seymour (1987, Table 1) and from our study when snakes are grouped according to his categories. As a percent of estimated (see above) total length, our data set does not indicate arboreal species to have the most anteriorly placed hearts. Therefore, we conclude that the discrepancy between our analyses and those of Seymour (1987) cannot be explained entirely by the difference in the measure of body size used.

Table 6: Heart position as a percentage of body length

\begin{tabular}{lccc}
\hline & \multicolumn{3}{l}{ Percentage of } \\
\cline { 2 - 4 } & $\begin{array}{l}\text { Total Length } \\
\text { (Seymour } \\
1987)\end{array}$ & $\begin{array}{l}\text { Estimated Total } \\
\text { Length (This } \\
\text { Study) }\end{array}$ & $\begin{array}{l}\text { Snout-Vent } \\
\text { Length (This } \\
\text { Study) }\end{array}$ \\
\hline Arboreal & $17.4(2.2)$ & $17.9(4.1)$ & $25.6(5.8)$ \\
Terrestrial & $18.8(3.2)$ & $15.6(2.9)$ & $19.4(3.7)$ \\
Semiaquatic & $22.7(4.8)$ & $15.7(2.3)$ & $19.4(2.8)$ \\
Fossorial & $23.6(7.9)$ & $17.6(2.9)$ & $21.8(3.5)$ \\
Viperidae & $33.3(4.5)$ & $25.2(3.8)$ & $31.9(4.2)$ \\
Aquatic & $33.4(5.7)$ & & \\
\hline
\end{tabular}

Note. Data are means, with SDs in parentheses. 


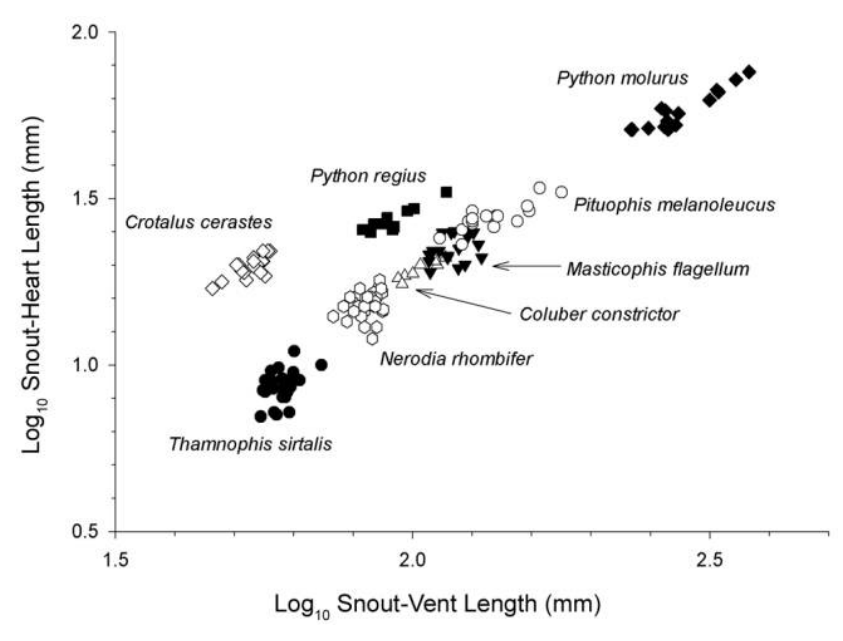

Figure 5. Illustration of the range of individual variation in snoutheart length in relation to snout-vent length for eight species in which we had at least 10 individuals per species. See "Discussion."

\section{Caveats}

A limitation of any comparative study based on data sets comprised of faunal surveys or literature reviews is that the various species are not measured under "common garden" conditions (Garland and Adolph 1991, 1994; Garland et al. 2005). Hence, it is possible that some of the differences we have observed among habitats or among clades might be reduced in magnitude if all animals had been raised under identical conditions or even housed under identical conditions for some weeks or months before measurement (see Garland and Adolph 1991 and references therein). On the other hand, it would not be possible to raise all species under identical conditions because, for example, they will not (voluntarily) eat exactly the same types of food. In addition, heart position can shift ontogenetically within a species (S. M. Secor, unpublished results), so variation in age among species in our sample would affect species differences to some extent.

Most of the species included here are represented by a single individual, which obviously introduces error when comparing species. To get some indication of how this "noise" may have influenced our comparison, in Figure 5 we show the relation between $\log _{10}$ SHL and $\log _{10}$ SVL for the eight species from the Secor sample that were represented by 10 or more nonjuvenile individuals (total $N=150$ ). As can be seen, the magnitude of the differences among some species in size-relative heart position is much larger than the range of variation observed within species. In general, noise introduced by small sample sizes should tend to reduce statistical power to detect effects of habitat, clade, etc. Given the several statistically significant effects we have detected (Table 3), this sampling variation was not large enough to obscure major results. An improvement for future analyses would be to fit regression models that explicitly account for the magnitude of within-species variation (Ives et al. 2007).

An additional caveat is that the taxa used in this study were different than those used in previous studies. In particular, only 12 of the 155 taxa used in this present study were also used in Seymour (1987; see Table 7). Moreover, 51 of our species (33\%) were South American xenodontines, a taxon lacking from Seymour's (1987) sample, whereas we lacked any fully aquatic snakes, such as hydrophiid sea snakes, which accounted for $17 \%$ of Seymour's sample. On the other hand, of the 14 families and subfamilies included in one or the other of the two studies, nine were included in both (Table 7).

A final limitation is that it is very difficult to quantify and categorize behavior - especially in broadly defined regimes such as "habitat" (e.g., see Jayne 1982). Very few snakes live strictly in an arboreal regime or a terrestrial regime (sea snakes and blind snakes being notable exceptions) but instead tend to exist at the borders of any given behavioral spectrum (e.g., primarily

Table 7: Unique and shared taxa between Seymour (1987) and this study

\begin{tabular}{llll}
\hline Shared Taxa & $\begin{array}{l}\text { Unique Taxa in } \\
\text { This Study }\end{array}$ & $\begin{array}{l}\text { Unique Taxa in } \\
\text { Seymour (1987) }\end{array}$ & Shared Species \\
\hline Boinae & & Acrochordidae & \\
Colubrinae & & & $\begin{array}{l}\text { Agkistrodon piscivorus } \\
\text { Arizona elegans }\end{array}$ \\
Crotalinae & Dipsadinae & & $\begin{array}{l}\text { Bothrops atrox } \\
\text { Diadophis punctatus } \\
\text { Elapinae }\end{array}$ \\
Erycinae & & Homalopsinae obsoleta & Lachesis muta \\
& & Hydrophiinae & Masticophis flagellum \\
& & & Nerodia sipedon \\
Natricinae & & & Nerodia taxispilota \\
Pythoninae & & & Pituophis melanoleucus \\
Typhlopidae & & & Thamnophis sirtalis \\
Viperinae & & & \\
& Xenodontinae & &
\end{tabular}

Note. See "Discussion." 
terrestrial but occasionally arboreal or primarily fossorial but occasionally terrestrial). Thus, categorization of habitat becomes particularly difficult for animals that can be classified a: semiarboreal or semifossorial. For our purposes, any snake that frequently assumes vertical postures, even if commonly founc in a terrestrial environment (e.g., Pantherophis), was considered arboreal, but one can see how tinkering with such a distinction could have important consequences for the results and con clusions of a study such as this. Note, however, that our data set does include some strictly arboreal species (e.g., Corallus sp., Atheris squamiger, Bothriopsis bilineata, Oxybelis sp., Im antodes cenchoa, etc.). Because we present all of the data analyzed here (App. A), it will be possible for future workers to try various recategorizations as field data become more avail able. In addition, future workers can incorporate additional ecological or behavioral predictors, such as diet (Hamptor $\rightarrow$ 2009).

\section{Acknowledgments}

We thank H. B. Lillywhite for helpful discussions. This study was supported by National Science Foundation grants DEB0416085 (D. N. Reznick, M. S. Springer, and T.G.) and IOS 0466139 (S.M.S.). A.S.A. and D.V.A. were supported by Fundação de Amparo à Pesquisa do Estado de São Paulo and Conselho Nacional de Desenvolvimento Científico e Tecnoló gico grants, and T.W. was supported by the Danish Research Council.

\section{Literature Cited}

$\rightarrow$ Badeer H.S. 1998. Anatomical position of heart in snakes with vertical orientation: a new hypothesis. Comp Biochem Phys $\rightarrow$ iol A 119:403-405.

$\rightarrow$ Blomberg S.P. and T. Garland Jr. 2002. Tempo and mode in evolution: phylogenetic inertia, adaptation and comparativi methods. J Evol Biol 15:899-910.

$\rightarrow$ Blomberg S.P., T. Garland Jr., and A.R. Ives. 2003. Testing for phylogenetic signal in comparative data: behavioral traits are more labile. Evolution 57:717-745.

$\rightarrow$ Buchwalter D.B., D.J. Cain, C.A. Martin, L. Xie, S.N. Luoma, and T. Garland Jr. 2008. Aquatic insect ecophysiological traits reveal phylogenetically based differences in dissolved cadmium susceptibility. Proc Natl Acad Sci USA 105:8321-8326 -

Buckner P., A. Quail, D. Cottee, and S. White. 1999. Venous hydrostatic indifference point as a marker of postnatal adaptation to orthostasis in swine. J Appl Physiol 87:882-888 -

Burnham K.P. and D.R. Anderson. 2002. Model Selection and Multi-model Inference: A Practical Information-Theoretic Approach. 2nd ed. Springer, New York.

Clark J.H., D.R. Hooker, and L.H. Weed. 1934. The hydrostatic factor in venous pressure measurements. Am J Physiol 109: 166-177.

Clobert J., T. Garland Jr., and R. Barbault. 1998. The evolution of demographic tactics in lizards: a test of some hypotheses concerning life history evolution. J Evol Biol 11:329-364.

$\rightarrow$ Cohn M.J. and C. Tickle. 1999. Developmental basis of limblessness and axial patterning in snakes. Nature 399:474-479.

$\rightarrow$ de Queiroz A. and J.A. Rodríguez-Robles. 2006. Historical contingency and animal diets: the origins of egg eating in snakes. Am Nat 167:684-694.

$\rightarrow$ Díaz-Uriarte R. and T. Garland Jr. 1998. Effects of branch length errors on the performance of phylogenetically independent contrasts. Syst Biol 47:654-672.

$\rightarrow$ Duncan R.P., D.M. Forsyth, and J. Hone. 2007. Testing the metabolic theory of ecology: allometric scaling exponents in mammals. Ecology 88:324-333.

$\rightarrow$ Felsenstein J. 1988. Phylogenies and quantitative characters. Annu Rev Ecol Syst 19:445-471.

Freckleton R.P., P.H. Harvey, and M. Pagel. 2002. Phylogenetic analysis and comparative data: a test and review of evidence. Am Nat 160:712-726.

$\rightarrow$ Garland T., Jr., and S.C. Adolph. 1991. Physiological differentiation of vertebrate populations. Annu Rev Ecol Syst 22: 193-228.

$\rightarrow$ 1994. Why not to do two-species comparative studies: limitations on inferring adaptation. Physiol Zool 67:797-828.

$\rightarrow$ Garland T., Jr., A.F. Bennett, and E.L. Rezende. 2005. Phylogenetic approaches in comparative physiology. J Exp Biol 208:3015-3035.

$\rightarrow$ Garland T., Jr., and P.A. Carter. 1994. Evolutionary physiology. Annu Rev Physiol 56:579-621.

$\rightarrow$ Garland T., Jr., A.W. Dickerman, C.M. Janis, and J.A. Jones. 1993. Phylogenetic analysis of covariance by computer simulation. Syst Biol 42:265-292.

$\rightarrow$ Garland T., Jr., P.H. Harvey, and A.R. Ives. 1992. Procedures for the analysis of comparative data using phylogenetically independent contrasts. Syst Biol 41:18-32.

Garland T., Jr., and A.R. Ives. 2000. Using the past to predict the present: confidence intervals for regression equations in phylogenetic comparative methods. Am Nat 155:346-364.

$\rightarrow$ Garland T., Jr., P.E. Midford, and A.R. Ives. 1999. An introduction to phylogenetically based statistical methods, with a new method for confidence intervals on ancestral values. Am Zool 39:374-388.

Gauer O.H. and H.L. Thron. 1965. Postural changes in the circulation. Pp. 2409-2439 in W.F. Hamilton and P. Dow, eds. Handbook of Physiology. Sec. 2. Circulation. Vol. 3. American Physiological Society, Washington, DC.

$\rightarrow$ Goldsmith S.K. 1984. Aspects of the natural history of the rough green snake, Opheodrys aestivius (Colubridae). Southwest Nat 29:445-452.

$\rightarrow$ Gould S.J. and R. Lewontin. 1979. The spandrels of San Marcos and the Panglossian paradigm: a critique of the adaptationist programme. Proc R Soc B 205:581-598.

$\rightarrow$ Grafen A. 1989. The phylogenetic regression. Philos Trans R Soc B 326:119-157.

Greene H.W. 1986. Diet and arboreality in the emerald monitor, Varanus prasinus, with comments on the study of adaptation. Fieldiana 31:1-12. 
1997. Snakes: The Evolution of Mystery in Nature. University of California Press, Berkeley.

Greene H.W. and R.W. McDiarmid. 2005. Wallace and Savage: heroes, theories and venomous snake mimicry. Pp. 190-208 in M.A. Donnelly, B.I. Crother, C. Guyer, M.H. Wake, and M.E. White, eds. Ecology and Evolution in the Tropics: A Herpetological Perspective. University of Chicago Press, Chicago.

$\rightarrow$ Guyer C. and M.A. Donnelly. 1990. Length-mass relationships among an assemblage of tropical snakes in Costa Rica. J Trof Ecol 6:65-76.

$\rightarrow$ Halsey L.G., P.J. Butler, and T.M. Blackburn. 2006. A phylogenetic analysis of the allometry of diving. Am Nat 167:276287.

Hampton P.M. 2009. Morphological and anatomical correlates to prey shape in snakes. Integr Comp Biol 49(online suppl. 1):e70.

Hargens A. 1991. Developmental adaptations to gravity in animals. NASA Technical Memorandum, 102228, unclassified.

$\rightarrow$ Hargens A.R., R.W. Millard, K.S. Pettersson, and K. Johansen. 1987. Gravitational haemodynamics and oedema prevention in the giraffe. Nature 329:59-60.

Harvey P.H. and M.D. Pagel. 1991. The Comparative Method in Evolutionary Biology. Oxford University Press, Oxford.

$\rightarrow$ Huey R.B., C.A. Deutsch, J.J. Tewksbury, L.J. Vitt, P.E. Hertz, H.J. Álvarez Pérez, and T. Garland Jr. 2009. Why tropical forest lizards are vulnerable to climate warming. Proc R Sor $\rightarrow$ B 276:1939-1948.

$\rightarrow$ Ives A.R., P.E. Midford, and T. Garland Jr. 2007. Within-specie: variation and measurement error in phylogenetic comparative methods. Syst Biol 56:252-270.

Jarvis S., D. Sheriff, and J. Pawelczyk. 2007. The volume indifferent point: an approach for explaining orthostatic in tolerance. FASEB J 21:750.10. (Abstr.)

$\rightarrow$ Jayne B.C. 1982. Comparative morphology of the semispinalis spinalis muscle of snakes and correlations with locomotion and constriction. J Morphol 172:83-96.

$\rightarrow$ Jeffery N., T.M. Ryan, and F. Spoor. 2008. The primate subarcuate fossa and its relationship to the semicircular canals. II. Adult interspecific variation. J Hum Evol 55:326-339.

$\rightarrow$ Lavin S.R., W.H. Karasov, A.R. Ives, K.M. Middleton, and T. Garland Jr. 2008. Morphometrics of the avian small intestint $\rightarrow$ compared with that of non-flying mammals: a phylogenetic approach. Physiol Biochem Zool 81:526-550.

$\rightarrow$ Lillywhite H.B. 1987. Circulatory adaptations of snakes to grav ity. Am Zool 27:81-95.

$\rightarrow \longrightarrow$. 1988. Snakes, blood circulation and gravity. Sci Am 256:92-98.

$\rightarrow-$ 1993. Subcutaneous compliance and gravitational adaptations in snakes. J Exp Zool 267:557-562.

$\rightarrow$. 1996. Gravity, blood circulation, and the adaptation of form and function in lower vertebrates. J Exp Zool 275: 217-225.

$\rightarrow$ Lillywhite H.B., R.E. Ballard, and A.R. Hargens. 1996a. Cardiovascular responses of semi-arboreal snakes to chronic, intermittent hypergravity. J Comp Physiol B 166:241-253. 1996b. Tolerance of snakes to hypergravity. Physiol Zool 69:239-303.

Lillywhite H.B. and R.W. Henderson. 1993. Behavioral and functional ecology of arboreal snakes. Pp. 1-48 in R.A. Seigel and J.T. Collins, eds. Snakes, Ecology and Behavior. McGrawHill, New York.

Manzani P.R. 1995. Açao da gravidade e sistema cardiovascular em serpentes: habito ou filogenia? Universidade Estadual Paulista, Rio Claro.

$\rightarrow$ Martins E.P. and T.F. Hansen. 1997. Phylogenies and the comparative method: a general approach to incorporating phylogenetic information into the analysis of interspecific data. Am Nat 149:646-667 (erratum, 153:448).

$\rightarrow$ Martins M., O.A.V. Marques, and I. Sazima. 2008. How to be arboreal and diurnal and still stay alive: microhabitat use, time of activity, and defense in Neotropical forest snakes. S Am J Herpetol 3:58-67.

Martins M. and M.E. Oliveira. 1998. Natural history of snakes in forests of the Manaus region, central Amazonia, Brazil. Herpetol Nat Hist 6:78-150.

Nilsson O., S. Booj, A. Dahlstrom, A.R. Hargens, R.W. Millard, and K.S. Pettersson. 1988. Sympathetic innervation of the cardiovascular system in the giraffe. Blood Vessels 25:299307.

Orzack S. and E. Sober, eds. 2001. Adaptationism and Optimality. Cambridge University Press, Cambridge.

Pagel M.D. 1992. A method for the analysis of comparative data. J Theor Biol 156:431-442.

Rayner J.M.V. 1985. Linear relations in biomechanics: the statistics of scaling functions. J Zool (Lond) 206:415-439.

Rose M. and G.V. Lauder. 1996. Adaptation. Academic Press, San Diego, CA.

$\rightarrow$ Seymour R.S. 1987. Scaling of cardiovascular physiology in snakes. Am Zool 27:97-109.

$\rightarrow$ Seymour R.S. and H.B. Lillywhite. 1976. Blood pressure in snakes from different habitats. Nature 264:664-667.

$\rightarrow$ Swanson D.L. and T. Garland Jr. 2009. The evolution of high summit metabolism and cold tolerance in birds and its impact on present-day distributions. Evolution 63:184-194.

Uetz P., J. Goll, and J. Hallermann. 2007. Die TIGR-Reptiliendatanbank. Elaphe 15:22-25.

Wagner E. 1886. Fortgesetzte Untersuchungen über den Einfluss der Schwere auf den Kreislauf. Arch Ges Physiol 39: 371.

$\rightarrow$ Warne R.W. and E.L. Charnov. 2008. Reproductive allometry and the size-number trade-off for lizards. Am Nat 172:E80E98.

$\rightarrow$ Warton D.I., I.J. Wright, D.S. Falster, and M. Westoby. 2006. Bivariate line-fitting methods for allometry. Biol Rev 81:259_ 291.

Williamson J.R., N.J. Vogler, and C. Kilo. 1971. Regional variations in the width of the basement membrane of muscle capillaries in man and giraffe. Am J Pathol 63:359-370.

Wright A.H. and A.A. Wright. 1957. Handbook of Snakes of the United States and Canada. Cornell University Press, Ithaca, NY. 